\title{
Shigella and Salmonella Infections are Associated with Markers of Environmental Enteropathy among Children under Five in Zambia
}

\section{Michelo Simuyandi ${ }^{1}$, Samuel Bosomprah ${ }^{1,2^{*}}$, Natasha Makabilo Laban ${ }^{1}$, Katayi Mwila-Kazimbaya ${ }^{1}$, Roma Chilengi ${ }^{1}$ and Caroline $\mathrm{C}^{\mathrm{C}}$ hisenga ${ }^{1}$}

${ }^{1}$ Centre for Infectious Disease Research in Zambia, Lusaka, Zambia

${ }^{2}$ Department of Biostatistics, School of Public Health, University of Ghana, Accra, Ghana

*Corresponding author: Samuel Bosomprah, Department of Biostatistics, School of Public Health, University of Ghana, Accra, Ghana, Tel: +260 968356711; E-mail: sbosomprah@gmail.com

Received date: May 30, 2019; Accepted date: June 22, 2019; Published date: July 01, 2019

Citation: Simuyandi M, Bosomprah S, Laban NM, Kazimbaya KM, Chilengi R, et al. (2019) Shigella and Salmonella Infections are Associated with Markers of Environmental Enteropathy among Children under Five in Zambia. Pediatric Infect Dis Vol.4 No.1:3.

Copyright: ${ }^{\odot} 2019$ Simuyandi M, et al. This is an open-access article distributed under the terms of the Creative Commons Attribution License, which permits unrestricted use, distribution, and reproduction in any medium, provided the original author and source are credited.

\section{Abstract}

Background: Environmental Enteropathy (EE) is a complex syndrome involving intestinal disorder which has been observed among children living in resource-poor settings. We investigated stored stool samples collected from children under-5 for relationships between specific enteric pathogens and levels of stool EE markers: Calprotectin (CALP), Alpha-1-Anti-Trypsin (AAT) and Myeloperoxidase (MPO).

Methods: We used clinical data and stored stool samples collected prior to, and during the early months of widespread rotavirus vaccine implementation in Lusaka between July 2012 and October 2013 in Lusaka, Zambia. Children were considered eligible if they presented to the facility with symptoms of moderate-to-severe diarrhoea defined by having one or more of the following signs or symptoms: dehydration evidenced by sunken eyes, loss of normal skin turgor, or requiring intravenous resuscitation; dysentery (diarrhoea with blood in stool); or hospitalisation. We randomly selected 320 stool samples from the pool of 2,050 for analysis. We tested for 15 enteric pathogens using the Luminex XTag GPP panel and used commercial ELISA kits to assess the presence and levels of three known fecal markers of EE. We derived an EE score using a weighted sum of CALP, AAT, and MPO and applied a multivariable linear regression model to identify enteric pathogens independently associated with EE score.

Results: After excluding insufficient samples (24) and those with missing clinical data (62), a total of the 234 were available for analysis. Of these, 114 (49\%) were female, 103 (44\%) were asymptomatic for diarrhoea, 181 (77\%) had two or more enteric pathogens detected in stool. Fifty-six percent $(131 / 234)$ children were positive for Rotavirus, 43.2\% (101/234) Enterotoxigenic E. coli, 36\% (86/234) Giardia, 35\% (82/234) Adenovirus and 34.2\% (80/234) had Shigella. The mean EE score was $5(\mathrm{SD}=2.25)$. The median concentration of MPO, CALP, and AAT were $2560.4 \mathrm{ng} / \mathrm{ml}$ (interquartile range $(\mathrm{IQR})=806.1,6522.9), 79.1 \mathrm{ng} / \mathrm{ml}$ $(\mathrm{IQR}=0,362.5)$, and $48.6 \mathrm{mg} / \mathrm{ml} \quad(\mathrm{IQR}=12.1, \quad 139.5)$ respectively. Shigella (coefficient $=0.92 ; 95 \% \mathrm{Cl}=(0.14,1.70)$; $\mathrm{p}=0.022)$ and Salmonella (coefficient $=0.83 ; 95 \% \mathrm{Cl}=(0.08$, 1.58); $p=0.030$ ) were independently associated with increase in mean $\mathrm{EE}$ score and together with $E$. coli accounted for $68.5 \%$ of the predicted variance in EE score.

Conclusions: Salmonella and Shigella were found to be associated with $\mathrm{EE}$ and together with E.coli accounted for over two-thirds of variability in the EE score.

Keywords: Fecal biomarkers; Environmental enteropathy; Salmonella and Shigella; Zambian children

\section{Introduction}

Environmental enteric dysfunction (EED) also known as environmental enteropathy (EE) is a complex syndrome involving an intestinal disorder which has been observed among children living in resource-poor settings characterised by poor sanitation and hygiene [1]. In the most affected regions, up to $75 \%$ prevalence has been reported, and the syndrome has also been linked to long term sequelae, which includes stunting [2], poor cognitive development, diminished oral vaccine effectiveness, delayed psychomotor development [3], and increased risk of cardiovascular disease later in life [4].

Repeated exposure to enteric pathogens through fecal contamination is postulated as the major risk factor for EE [1]. The syndrome results in morphological changes in the gut, including intestinal epithelial damage, increased permeability, and microbial translocation into the lamina propria [5-7]. Recent studies have shown that some specific enteric infections such as Shigella and Enterotoxigenic E. coli (ETEC) are especially associated with EE [8] while Entero-aggregative E. coli (EAEC), Campylobacter and Giardia are associated with gut inflammation markers of $\mathrm{EE}[9,10]$.

Diagnosis of EE is either through (i) an endoscopy or (ii) biopsy, both of which are invasive and difficult to perform in lowresource settings [11]. The non-invasive urine test, Lactulose- 
Mannitol ratio permeability test, is an alternative but requires a 5 -hour urine collection which is very cumbersome and renders it unattractive [4] and difficult to implement in a resource-limited setting. Myeloperoxidase (MPO), Calprotectin (CALP) and Alpha-1 Anti-Trypsin (AAT) are potential proxy fecal markers for the evaluation of gut barrier function and damage and also demonstrate both the presence and extent of EE [12-14]. MPO is a lysosomal protein contained within primary granules that are released into the gut lumen by activated neutrophils and other phagocytes during acute inflammation [15].

Higher concentrations of MPO in the stool suggest lymphocytic infiltration of the lamina propria [16]. AAT on the other hand is a protease inhibitor abundant in serum which appears to protect cells from inflammatory proteases secreted by neutrophils and macrophages. It is a large, polar molecule such that it does not cross the luminal barrier unless there is significantly aberrant permeability $[17,18]$. AAT is a useful marker for intestinal permeability and protein-losing enteropathy [19]. CALP accounts for more than half of the total soluble proteins in the cytosolic fraction of neutrophil granulocytes $[20,21]$. Based on several studies that have shown increased levels of fecal CALP in inflammatory bowel disease, it is now used as a marker of gastrointestinal inflammation in humans [14].

Understanding risk factors and consequences of EE is critical for the development of effective interventions for prevention and treatment and subsequently the improvement of child health and future quality of life. In this study, we investigated stored stool samples collected from children under-5 presenting with Moderate-to-Severe Diarrhoea (MSD) at health facilities for relationships between specific enteric pathogens and levels of stool EE markers: MPO, AAT, and CALP. We hypothesised that diarrhoeagenic enteric pathogens are important predictors of EE.

\section{Methods}

\section{Study design and participants}

We used clinical data and stored stool samples collected prior to, and during the early months of widespread rotavirus vaccine implementation in Lusaka between July 2012 and October 2013 in Lusaka, Zambia. The design of the study was described in detail elsewhere [22,23], but briefly, children were considered eligible if they presented to the facility with symptoms of having one or more of the following signs or symptoms: dehydration evidenced by sunken eyes, loss of normal skin turgor, or requiring intravenous resuscitation; dysentery (diarrhoea with blood in stool); or hospitalization. For each child enrolled, clinical and socio-demographic data and a raw stool sample were collected after written informed consent was obtained from the parent or legal guardian. The stool samples remaining after the conclusion of the original study were stored at $-80^{\circ} \mathrm{C}$. In the present study, we selected 320 stored stool samples using a simple random sampling algorithm implemented in Stata $15 \mathrm{MP}$ (StataCorp, College Station, TX, USA). We excluded samples with duplicates ID as well as those insufficient for EE biomarker testing.

\section{Laboratory procedures}

Fecal biomarkers and reagent preparations: We used commercial ELISA kits (Immundiagnostik AG, Bensheim Germany) to assess the presence and level of EE markers in the stool. In brief, we ran $15 \mathrm{mg}$ of stool or $0.015 \mathrm{mls}$ (if the stool was watery) per test. The initial dilutions for both CALP and AAT was $1: 100 \mu \mathrm{g} / \mathrm{L}$, while that of MPO was $1: 500 \mathrm{ng} / \mathrm{L}$. When preparing wash buffer, the stock solution was placed in a water bath at $37^{\circ} \mathrm{C}$ for $10 \mathrm{~min}$ to dissolve the crystals, after which it was diluted 1:10 times with ultrapure water before use. The rest of the standards and reagents were prepared according to the manufacturer's instructions two hours before the experiment could be run and were equally kept at $4^{\circ} \mathrm{C}$ when not in use for up to 12 hours.

ELISA assay procedure: The ELISA plate was washed using the wash buffer 2 times before labeling the portions for adding standards, samples, and controls. The wash buffer was then discarded and the inverted plate dried by tapping it on absorbent filter papers. This was followed by the addition 100 $\mu \mathrm{ls}$ of the prepared standards, samples and controls, all in duplicates and equal amounts. The plates were sealed with a cover and incubated at room temperature on a horizontal shaker for 1 hour. Following incubation, the cover was removed and the contents of each well were discarded and the plate was washed five [5] times with $250 \mu \mathrm{ls}$ wash buffer and inverted plate dried by tapping the plate on the absorbent filter without letting the wells to dry completely. Using the multichannel pipette, $100 \mu \mathrm{L}$ of biotinylated detection antibody working solution was added into the bottom of the wells (standard, test sample, and control) without touching the side wall. The plate was sealed again and incubated for at $37^{\circ} \mathrm{C}$ for 1 hour on a horizontal shaker. After incubation, the seal was removed and the plates washed 5 times again with wash buffer and inverted plate dried by tapping the plate on the absorbent filter. Then $100 \mu \mathrm{L}$ of conjugate antibody was added into the bottom of the wells (standard, test sample, and control) without touching the side wall.

The plate was sealed again and incubated for at $37^{\circ} \mathrm{C}$ for 1 hour on a horizontal shaker. After this step, the 5 times wash step was repeated and then, $100 \mu$ of $\operatorname{TMB}\left(3,3^{\prime}, 5,5^{\prime}\right.$ tetramethylbenzidine) substrate solution was then added into each well, covered the plate and incubated at room temperature 15 minutes. The stop solution $(100 \mu \mathrm{l})$ was added to each well and mixed thoroughly. At this stage, plates were immediately read at the optic density absorbance of $450 \mathrm{~nm}$ in a micro plate reader. Depending on the marker to be read, the incubation time and temperature were adjusted accordingly as per kit manufacturer instructions.

Result calculation: For standard curve generation, we used a 4-parameter logistic (4-PL) model because the antibody and analyte (standard) in the ELISA assay exhibits a sigmoidal relationship with concentration. The 4-PL model was used since it optimises accuracy and precision over the maximum usable calibration range [24]. We then used the resultant standard curves to calculate the concentrations of the samples using the mean optical density values for each sample. 
Enteric pathogens: We used the qualitative, multiplex, Polymerase Chain Reaction (PCR) based Luminex $x$-TAG ${ }^{\circledR}$ gastrointestinal pathogen panel (GPP) (Luminex Corporation, Austin TX, USA) to detect 15 enteric pathogens (i.e. viruses, bacteria, and protozoa) as reported elsewhere [23]. The assay procedure described in the cited paper was followed through step by step in order to profile the enteric pathogens.

\section{Sample size calculation}

We assumed that the squared multiple correlation coefficient $\left(\rho^{2}\right)$ in the general population was 0.11 . The primary analysis was tests of regression coefficients in a multivariable linear regression model of 9 independent variables with positive associations with EE score. A sample size of 230 achieves $80 \%$ power to detect a partial $\rho^{2}$ of at least 0.110 attributed to 9 independent variable(s) when the significance level (alpha) is 0.050 and the actual value of $\rho^{2}$ is 0.230 . The influence of an additional 9 independent variable(s) was removed.

\section{Statistical analysis}

The primary outcome was environmental enteropathy score (EE score). The exposures of interest were having MSD and the presence of enteric pathogens in the stool. We calculated the EE score as the weighted sum of categories of MPO, AAT, and CALP using weights as previously published [25]. We defined the categories based on the distribution of the data as 0 " $<=25^{\text {th }}$ percentile" ; 1 " $>25^{\text {th }}$ to $<75^{\text {th }}$ percentiles "; and 2 " $>=75^{\text {th }}$ percentile" such that the range of EE score was 0 to 10 . The higher the score the higher the EE severity.

EE score $=2 * \mathrm{MPO}+2 * \mathrm{AAT}+1 * \mathrm{CALP}$

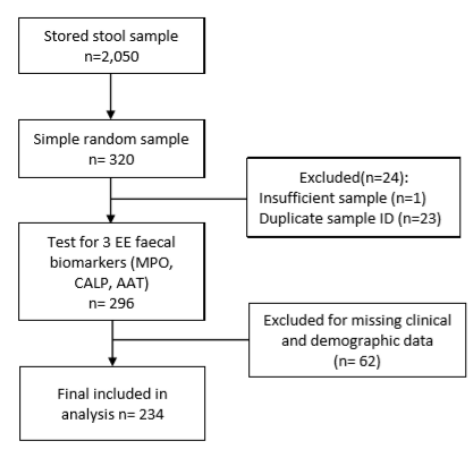

Figure 1: Study flow diagram.

We examined the distribution of the fecal markers and $E E$ score using box plots and summarised the EE score using means and standard deviation. Since EE scores were normally and independently distributed, we used a linear regression model to investigate the independent association of enteric pathogens with EE score adjusting for the number of enteric pathogens, age of the child, sex, and caregiver's level of education. We considered $p$-values $<0.05$ as statistically significant. In the multivariable model, we included only enteric pathogens that increased the mean EE score.

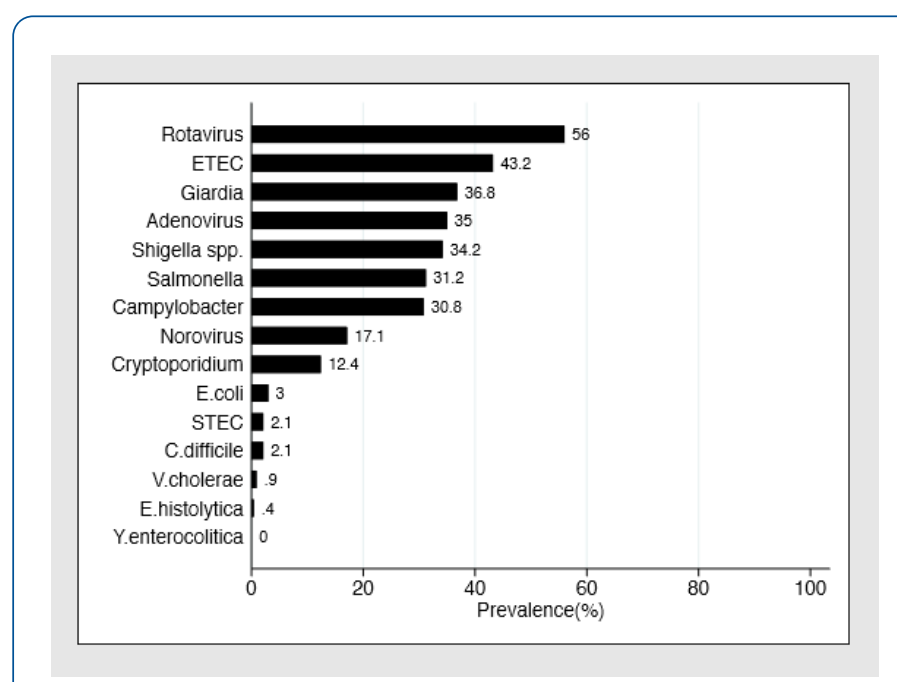

Figure 2: Prevalence of 15 enteric pathogens using Luminex XTag Gastrointestinal pathogen panel.

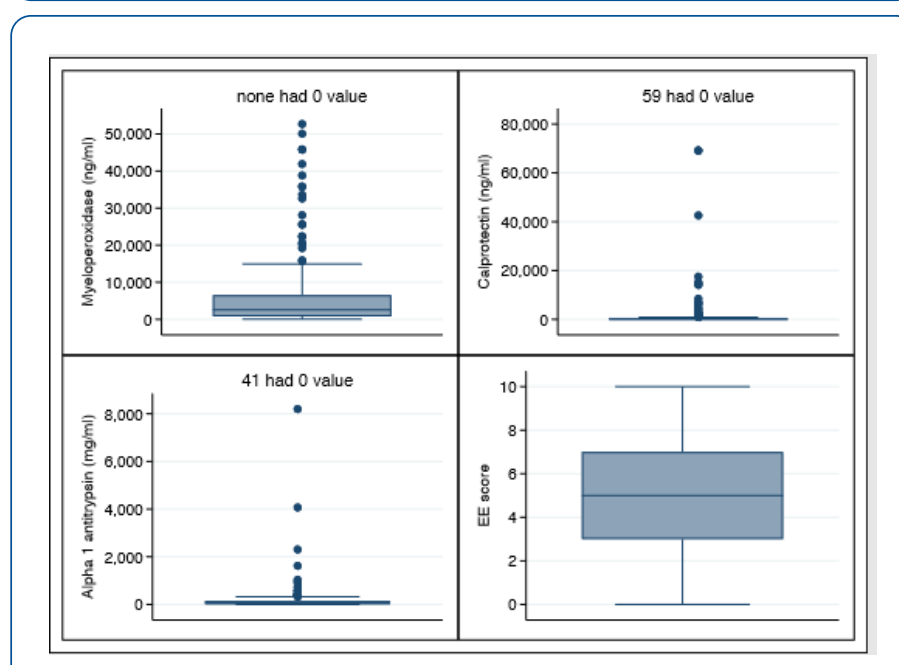

Figure 3: Distribution of stool biomarkers of environmental enteropathy and EE score across the study population. Clockwise: Myeloperoxidase, Calprotectin, EE score, and Alpha 1 antitrypsin.

We used dominance analysis [26] to estimate the relative importance of the enteric pathogens in the statistical model. For ease of interpretation, we reported the general dominance statistics which was derived as a weighted average marginal contribution to the overall fit statistic and R-squared, an enteric pathogen makes across the statistical models. Similarly, we used a generalised linear model with Gaussian-family and log-link to explore the independent association of enteric pathogens with MPO concentration. All analyses were performed using Stata 15 MP (StataCorp, College Station, T exas, USA). 
Table 1: Distribution of stool biomarkers of environmental enteropathy by social demographics, Lusaka.

\begin{tabular}{|c|c|c|c|c|c|}
\hline Characteristics & $\begin{array}{l}\text { Number of Infants } \\
\text { (\% of total) }\end{array}$ & \multicolumn{3}{|c|}{$n(\%)>75^{\text {th }}$ percentile } & $\begin{array}{l}\text { EED score*; mean } \\
\text { (SD) }\end{array}$ \\
\hline \multicolumn{6}{|c|}{ Age of child (months) } \\
\hline Median (IQR) & \multicolumn{2}{|l|}{$15.6(8.4,25.2)$} & & & \\
\hline $12-23$ & $74(32)$ & $22(30)$ & $14(19)$ & $23(31)$ & $4.95(2.47)$ \\
\hline $24-59$ & $71(30)$ & $9(13)$ & $13(18)$ & $17(24)$ & $4.70(1.99)$ \\
\hline \multicolumn{6}{|l|}{ Sex of child } \\
\hline \multicolumn{6}{|c|}{ Mother's education } \\
\hline Primary & $106(45)$ & $33(31)$ & $32(30)$ & $26(25)$ & $5.21(2.42)$ \\
\hline Secondary & $106(45)$ & $23(22)$ & $22(21)$ & $32(30)$ & $5.00(2.07)$ \\
\hline Higher & $9(4)$ & $1(11)$ & $1(11)$ & $0(0)$ & $4.11(1.54)$ \\
\hline \multicolumn{6}{|l|}{ Diarrhoea } \\
\hline Symptomatic & $131(56)$ & $30(23)$ & $35(27)$ & $41(31)$ & $5.22(2.11)$ \\
\hline Asymptomatic & $103(44)$ & $29(28)$ & $24(23)$ & $18(17)$ & $4.72(2.40)$ \\
\hline \multicolumn{6}{|c|}{ Number of siblings } \\
\hline 0 & $29(12)$ & $11(38)$ & $8(28)$ & $4(14)$ & $4.83(2.74)$ \\
\hline 1 & $38(16)$ & $8(21)$ & $12(32)$ & $6(16)$ & $4.74(2.14)$ \\
\hline 2 & $47(20)$ & $12(26)$ & $11(23)$ & $12(26)$ & $4.94(2.37)$ \\
\hline 3 & $53(23)$ & $14(26)$ & $12(23)$ & $15(28)$ & $5.09(2.22)$ \\
\hline 4 & $31(13)$ & $10(32)$ & $8(26)$ & $9(29)$ & $5.03(2.15)$ \\
\hline 5 & $23(10)$ & $4(17)$ & $5(22)$ & $8(35)$ & $5.04(2.33)$ \\
\hline $6+$ & $27(12)$ & $7(26)$ & $6(22)$ & $7(26)$ & $5.26(2.35)$ \\
\hline Total & $234(100)$ & $59(25)$ & $59(25)$ & $59(25)$ & $5(2.25)$ \\
\hline
\end{tabular}

\section{Ethical approval}

The University of Zambia Biomedical Research Ethics Committee and the University of North Carolina at Chapel Hill Institutional Review Board reviewed and approved the study.

The Zambian Ministry of Health provided the authorisation to conduct the research. Caregivers of participants provided written informed consent prior to initiation of study procedures for each enrolled child.

\section{Results}

\section{Characteristics of participants}

A total of 320 stool samples were randomly selected from 2,050 stored stool samples. Of the 320 selected samples, 24 were excluded due $((i)$ to insufficient samples $(n=1)$, and (ii) duplicate samples $(n=23)$ ), leaving a total of 296 stool samples available for EE biomarkers testing (Figure 1). Of the 296 samples, 62 were excluded due to missing clinical and 
demographic data, leaving a total of 234 with complete clinical and demographic data for analysis (Figure 1). Of the 234, 114 (49\%) were female, 103 (44\%) were asymptomatic for diarrohea, $181(77 \%)$ had two or more enteric pathogens detected in stool. The median age of children was 15.6 months (IQR=8.4, 25.2) with $88(38 \%)$ being infants (Table 1).

\section{Enteric pathogen prevalence}

The six (6) most common pathogens detected among children in this study were; Rotavirus (56\%), Enterotoxigenic E. coli (43.2\%), Salmonella (38.4\%), Giardia (36.8\%), Adenovirus (35\%), and Shigella (34.2\%) respectively (Figure 2 ).

\section{Fecal biomarkers of EE}

The mean EE score was $5 \quad(S D=2.25)$. The median concentration of MPO, CALP, and AAT were $2560.4 \mathrm{ng} / \mathrm{ml}$ (interquartile range $(I Q R)=806.1,6522.9), 79.1 \mathrm{ng} / \mathrm{ml}(I Q R=0$, 362.5), and 48.6 mg/ $\mathrm{ml}(\mathrm{IQR}=12.1,139.5)$ respectively (Figure 3).When stratified by social demographic status, children $<12$ months had a higher mean EE score $(5.3 \pm 2.42)$ compared to those between 12-23 months and those between 24-59 months, those with primary education had a higher mean EE score compare to those who had attained secondary even though the differences were not statistically significant (Table 1).

\section{Independent association of enteric pathogens with EE score}

In a multivariable linear regression model, we found evidence at a $5 \%$ level of significance that Shigella and Salmonella were independently associated with an increase in mean EE score (Table 2). Specifically, the presence of Shigella in stool was associated with an 0.92 unit increase in $E E$ score (coefficient $=0.92 ; \quad 95 \% \mathrm{Cl}=(0.14,1.70) ; \mathrm{p}=0.022$ ) while the presence of Salmonella was associated with an 0.83 unit increase in EE score (coefficient $=0.83 ; 95 \% \mathrm{Cl}=(0.08,1.58)$; $\mathrm{p}=0.030$ ) (Table 2). We also found borderline evidence that rotavirus (coefficient $=0.67 ; 95 \% \mathrm{Cl}=(-0.08,1.42) ; \mathrm{p}=0.081)$, E.coli (coefficient=1.59; $95 \% \mathrm{Cl}=(-0.24, \quad 3.42) ; \quad \mathrm{p}=0.088)$, and Cryptosporidium (coefficient $=0.92 ; \quad 95 \% \mathrm{Cl}=(-0.11, \quad 1.940$; $\mathrm{p}=0.078$ ) were independently associated with increase in mean EE score (Table 2). The association was even stronger in respect of Shigella, E. coli 0157 and, geometric mean increases in Myeloperoxidase (Table 2).

In the primary outcome of interest, EE score, we found that Salmonella, Shigella, and E. coli 0157 were the top 3 most ranked among the nine enteric pathogens included in the statistical model, and these accounted for $68.5 \%$ of the predicted variance $(29.7 \%, 22.5 \%$, and $16.0 \%$ respectively) (Table 2).

Table 2: Presence of enteric pathogens independently associated with EE score among children under 5 ( $n=233$ ).

\begin{tabular}{|c|c|c|c|c|}
\hline \multirow[b]{2}{*}{ Enteric pathogens } & \multirow{2}{*}{$\begin{array}{l}\text { Myeloperoxidase }(\mathrm{ng} / \mathrm{ml}) \\
{ }^{2} \text { Geometric mean ratio }(95 \% \mathrm{Cl}) \text {; p-value }\end{array}$} & \multicolumn{3}{|c|}{ EED score ${ }^{* *}$} \\
\hline & & ${ }^{1}$ Coefficient $(95 \% \mathrm{Cl}) ; \mathrm{p}$-value & $\begin{array}{l}\text { Standardised dominance } \\
\text { statistic }\end{array}$ & Rank \\
\hline \multicolumn{5}{|l|}{ Virus } \\
\hline Adenovirus & $1.24(0.74,2.07) ; 0.408$ & $0.41(-0.35,1.18) ; 0.289$ & 0.0132 & 7 \\
\hline Rotavirus & $0.83(0.49,1.38) ; 0.465$ & $0.67(-0.08,1.42) ; 0.081$ & 0.1256 & 5 \\
\hline \multicolumn{5}{|l|}{ Bacteria } \\
\hline C. difficile & $0.02(0.00,1822.33) ; 0.497$ & $0.78(-1.28,2.84) ; 0.457$ & 0.0266 & 6 \\
\hline E. coli 0157 & $3.78(2.41,5.93) ;<0.0001$ & $1.59(-0.24,3.42) ; 0.088$ & 0.1598 & 3 \\
\hline Enterotoxigenic E. coli & $0.78(0.50,1.21) ; 0.263$ & $0.43(-0.31,1.18) ; 0.250$ & 0.0086 & 8 \\
\hline Salmonella, spp. & $0.66(0.38,1.14) ; 0.136$ & $0.83(0.08,1.58) ; 0.030$ & 0.2971 & 1 \\
\hline Shigella spp. & $2.19(1.38,3.47) ; 0.001$ & $0.92(0.14,1.70) ; 0.022$ & 0.2248 & 2 \\
\hline \multicolumn{5}{|l|}{ Protozoa } \\
\hline Cryptosporidium & $0.50(0.17,1.44) ; 0.196$ & $0.92(-0.11,1.940) ; 0.078$ & 0.1436 & 4 \\
\hline E. histolytica & $5.65(1.31,24.30) ; 0.020$ & $0.47(-4.01,4.96) ; 0.835$ & 0.0008 & 9 \\
\hline \multicolumn{5}{|c|}{$\begin{array}{l}{ }^{1} \text { Coefficients were estimated using a linear regression model; }{ }^{2} \text { estimated using log-normal regression model. All estimates were adjusted for the number of enteric } \\
\text { pathogen infections (count); age of child in months (categorical); presence of enteric pathogens (binary); sex (binary); and caregiver's education (categorical) }{ }^{* *} E E \text { score } \\
\text { is a weighted sum of myeloperoxidase (MPO), alpha-1 anti-trypsin (AAT), and calprotectin (CALP) [25] }\end{array}$} \\
\hline
\end{tabular}

\section{Discussion}

In this paper, we report an association between enteric pathogen infection and EE score (measured using fecal biomarkers MPO, CALP and AAT) in Zambian children under 5 years. We found that Shigella and Salmonella were both independently associated with an increase in the mean EE score. These findings are consistent with the assertion that exposure to enteric pathogens can lead to EE $[14,27]$. We also found that Shigella and E. coli 0157 infection were strongly associated with 
an increase in geometric mean Myeloperoxidase. Other studies that have reported similar associations include George and colleagues who reported that children who had enterotoxigenic E. coli (ETEC) infection had significantly higher calprotectin concentrations; Kosek and colleagues showed that Campylobacter and enteroaggregative $E$. coli (EAEC) were associated with higher concentrations of MPO and AAT [27]. Rogawski and colleagues reported that the presence of Enteroaggregative E. coli (EAEC) and Campylobacter was associated with concentrations of MPO [10]. It is worth noting here that the enteric bacteria, which we reported to have an association with EE as well as the selected markers, are all enteroinvasive in nature or cause extensive mucosal disruption [7]. We also found that Salmonella, Shigella and E. coli had the largest contributions to the EE score. This observation could imply that early exposure to these pathogens may impact on the development of $E E$ and further research is needed to validate this observation. Identifying pathogens which are key drivers of EE development would help with interventions to prevent $E E$ in under 5 children. This observation is consistent with the common assertion that invasive pathogens could be contributing to EE [7].

The median AAT and CALP levels are lower in our population compared to other populations in LMICs such as Bangladesh [28]. In that study, younger infants from age one month were studied and most of these invariably were breastfed and this could explain the difference owing to protective benefits from breast milk. It would be important for future studies to monitor the potential increased risk with age and to verify whether, in fact, breast milk renders some protection.

Our results could be affected by several factors, some which include, (i) the study is the cross-sectional design with which we cannot demonstrate the causality; (ii) our study only focused on stool samples, and we did not have blood samples which could provide additional immunological information regarding serological markers and the immune status or response to these infections; we did not adjust for water sanitation and hygiene (WASH) status for which we had no data on exposure status. However, our study also had some strength: To the best of our knowledge, this is the first study to apply dominance analysis to statistical modeling of EE score-the result of which showed that $68.5 \%$ of the predicted variance in EE score was accounted for by Salmonella, Shigella and E.coli 0157.

These data confirm that invasive enteropathogens do induce and enhance EE. In addition, enteric infections are associated with other long-term sequelae such as (i) response to vaccination, (ii) child growth, (iii) cognitive development, and even later life (iv) obesity, (v) diabetes, and (vi) metabolic syndrome [29]. This is an important public health issue to LMIC settings where the WASH status is unlikely to improve generally in the immediate future. They reinforce the benefits from exclusive breastfeeding in early infancy and further underpin the need for vaccines against these pathogens.

\section{Conclusion}

Salmonella and Shigella were found to be associated with EE and together with $E$. coli accounted for over two-thirds of variability in EE score. While our study was not originally designed to address this issue, we have provided a firm basis for specific hypothesis-driven future studies. When validated, these biomarkers can be used to identify children at risk of EE for a targeted incremental nutritional supplement, especially where resources are limited to roll out interventions to the general population.

\section{Acknowledgment}

The authors thank the participants and the CIDRZ staff on the ACADEMIC study for their important contributions. The authors also thank the Biomedical Science students of the University of Zambia, College of Health Sciences (Malumbe Michelo, Lunenge Kasukumya, and Diana Zulu) for their help with the experimental work.

\section{Conflict of Interest}

All authors declared that they have no conflict of interest.

\section{Financial Disclosure}

Evaluation of the EE markers was financially supported by SHARE, a DFID funded consortium through the London School of Hygiene and Tropical Medicine. Support for the parent study was obtained from the Bill and Melinda Gates Foundation.

\section{Author Contribution}

- Michelo Simuyandi: Conceptualization; Investigation; Writing review and editing

- Samuel Bosomprah: Formal analysis; Methodology; Writingoriginal draft; Writing-review and editing

- Natasha Makabilo Laban: Data curation; Investigation; Writing-review and editing

- Katayi Mwila-Kazimbaya: Data curation; Investigation; Writingreview and editing

- Roma Chilengi: Conceptualization; funding acquisition; resources, supervision; Writing review and editing

- Caroline Cleopatra Chisenga: Data curation; Investigation; writing, review and editing

\section{Data Availability}

Data will be made available to any interested researchers upon request. The CIDRZ Ethics and Compliance Committee is responsible for approving such request. To request data access, one must write to the Committee chair/Chief Scientific Officer, Dr. Roma Chilengi, (Roma.Chilengi@cidrz.org) or the Secretary to the Committee/Head of Research Operations, Ms. Hope Mwanyungwi (Hope.Mwanyungwi@cidrz.org) through the corresponding author (Samuel Bosomprah, Ph.D.) mentioning the intended use for the data. The corresponding author will then facilitate express authorisation to release the data as requested. Dataset request must include contact information, a 
research project title, and a description of the analysis being proposed as well as the format it is expected. The requested data should only be used for the purposes related to the original research or study. The CIDRZ Ethics and Compliance Committee will normally review all data requests within 48-72 hours (Monday-Friday), and provide notification if access has been granted or additional project information is needed before access can be granted.

\section{References}

1. Watanabe K, Petri WA (2016) Environmental enteropathy: elusive but significant subclinical abnormalities in developing countries. EBioMedicine 10: 25-32.

2. Keusch GT, Denno DM, Black RE, Duggan C, Guerrant RL, et al. (2014) Environmental enteric dysfunction: pathogenesis, diagnosis, and clinical consequences. Clin Infect Dis 59: 207-212.

3. Lagos R, Fasano A, Wasserman SS, Prado V, Martin OS, et al. (1999) Effect of small bowel bacterial overgrowth on the immunogenicity of single-dose live oral cholera vaccine CVD 103HgR. J Infect Dis 180: 1709-1712.

4. Lee GO, Olortegui MP, Salas MS, Yori PP, Trigoso DR, et al. (2017) Environmental enteropathy is associated with cardiometabolic risk factors in Peruvian children. J Dev Orig Health Dis 8: 337-348.

5. Harper KM, Mutasa M, Prendergast AJ, Humphrey J, Manges AR (2018) Environmental enteric dysfunction pathways and child stunting: A systematic review. PLoS Negl Trop Dis 12: 6205.

6. Korpe PS, Petri WA (2012) Environmental enteropathy: critical implications of a poorly understood condition. Trends Mol Med 18: 328-336.

7. Kosek MN, Ahmed T, Bhutta Z, Caulfield L, Guerrant R, et al. (2017) Causal pathways from enteropathogens to environmental enteropathy: findings from the MAL-ED birth cohort study. EBio Medicine 18: 109-117.

8. George CM, Burrowes V, Perin J, Oldja L, Biswas S, et al. (2018) Enteric infections in young children are associated with environmental enteropathy and impaired growth. Trop Med Int Heal 23: 26-33.

9. Campbell DI, McPhail G, Lunn PG, Elia M, Jeffries DJ (2004) Intestinal inflammation measured by fecal neopterin in Gambian children with enteropathy: association with growth failure, Giardia lamblia, and intestinal permeability. J Pediatr Gastroenterol Nutr 39: 153-157.

10. Rogawski ET, Guerrant RL, Havt A, Lima IF, Medeiros, et al. (2017) Epidemiology of enteroaggregative Escherichia coli infections and associated outcomes in the MAL-ED birth cohort. PLoS Negl Trop Dis 11: 5798.

11. Crane RJ, Jones KD, Berkley JA (2015) Environmental enteric dysfunction: an overview. Food Nutr Bull 36: 76-87.

12. Colston JM, Peñataro YP, Colantuoni E, Moulton LH, Ambikapath $\mathrm{R}$, et al. (2017) A methodologic framework for modeling and assessing biomarkers of environmental enteropathy as predictors of growth in infants: an example from a Peruvian birth cohort. Am J Clin Nutr 106: 245-255.
13. De Serres F, Blanco I, (2014) Role of alpha-1 antitrypsin in human health and disease. J Intern Med 276: 311-335.

14. Naylor C, Lu M, Haque R, Mondal D, Buonomo E, et al. (2015) Environmental enteropathy, oral vaccine failure and growth faltering in infants in Bangladesh. EBioMedicine 2: 1759-1766.

15. Wagner M, Peterson CG, Ridefelt P, Sangfelt P, Carlson M (2008) Fecal markers of inflammation used as surrogate markers for treatment outcome in relapsing inflammatory bowel disease. World J Gastroenterol 14: 5584.

16. Keusch GT, Denno DM, Black RE, Duggan C, Guerrant RL, et al. (2014) Environmental enteric dysfunction: pathogenesis, diagnosis, and clinical consequences. Clin Infect Dis 59: 207-212.

17. Hill RE, Comm B, Hercz A, Corey ML, Gilday DL, et al. (1981) Fecal clearance of $\alpha 1$-antitrypsin: a reliable measure of enteric protein loss in children. J Pediatr 99: 416-418.

18. Karbach U, Ewe K, Bodenstein H (1983) Alpha 1-antitrypsin, a reliable endogenous marker for intestinal protein loss and its application in patients with Crohn's disease. Gut 24: 718-723.

19. Arndt MB, Richardson BA, Ahmed T, Mahfuz M, Haque R, et al (2016) Fecal markers of environmental enteropathy and subsequent growth in Bangladeshi children. Am J Trop Med Hyg 95: 694-701.

20. Gupta RC, (2019) Biomarkers in Toxicology. Elsevier Ed.

21. https://www.sciencedirect.com/book/9780702051401/clinicalbiochemistry-metabolic-and-clinical-aspects

22. Beres LK, Tate JE, Njobvu L, Chibwe B, Rudd C, et al. (2016) A preliminary assessment of rotavirus vaccine effectiveness in Zambia. Clin Infect Dis 62: 175-S182.

23. Chisenga CC, Bosomprah S, Laban NM, Mwila-Kazimbaya K, Mwaba J (2018) Aetiology of diarrhoea in children under five in Zambia. Pediatr Infect Dis Open Access 3: 1-6.

24. Findlay JW, Dillard RF (2007) Appropriate calibration curve fitting in ligand binding assays. AAPS J 9: 60-267.

25. Kosek M, Haque R, Lima A, Babji S, Shrestha S, et al. (2013) Fecal markers of intestinal inflammation and permeability associated with the subsequent acquisition of linear growth deficits in infants. Am J Trop Med Hyg 88: 390-396.

26. Azen R, Traxel N (2009) Using dominance analysis to determine predictor importance in logistic regression. J Educ Behav Stat 34: 319-347.

27. Kosek MN, Ahmed T, Bhutta Z, Caulfield L, Guerrant R, et al. (2017) Causal pathways from enteropathogens to environmental enteropathy: findings from the MAL-ED birth cohort study. EBioMedicine 18: 109-117.

28. McCormick BJ, Lee GO, Seidman JC, Haque R, Mondal D, et al. (2017) Dynamics and trends in fecal biomarkers of gut function in children from 1-24 months in the MAL-ED study. Am J Trop Med Hyg 96: 465-472.

29. Rogawski ET, Guerrant RL (2017) The burden of enteropathy and "subclinical" infections. Pediatr Clin 64: 815-836. 